\title{
Predictors of atrial rhythm after atrioventricular node ablation for the treatment of paroxysmal atrial arrhythmias
}

\author{
G M Gribbin, J P Bourke, J M McComb
}

\begin{abstract}
Objective-To assess the natural history of the atrial rhythm of patients with paroxysmal atrial arrhythmias undergoing atrioventricular node ablation and permanent pacemaker implantation. Design and setting-A retrospective cohort study of consecutive patients identified from the pacemaker database and electrophysiology records of a tertiary referral hospital.
\end{abstract}

Patients-62 consecutive patients with paroxysmal atrial arrhythmias undergoing atrioventricular node ablation and permanent pacemaker implantation between 1988 and July 1996.

Main outcome measures-(1) Atrial rhythm on final follow up ECG, classified as either ordered (sinus rhythm or atrial pacing) or disordered (atrial fibrillation, atrial flutter or atrial tachycardia). (2) Chronic atrial fibrillation, defined as a disordered rhythm on two consecutive ECGs (or throughout a 24 hour Holter recording) with no ordered rhythm subsequently documented.

Results-Survival analysis showed that $75 \%$ of patients progressed to chronic atrial fibrillation by 2584 days (86 months). On multiple logistic regression analysis a history of electrical cardioversion, increasing patient age, and VVI pacing were associated with the development of chronic atrial fibrillation. A history of electrical cardioversion and increasing patient age were associated with a disordered atrial rhythm on the final follow up ECG.

Conclusions-Patients with paroxysmal atrial arrhythmias are at high risk of developing chronic atrial fibrillation. A history of direct current cardioversion,

Accepted for publication 3 February 1998

Table 1 Baseline characteristics of the 62 patients

Mean (SD) age

Sex

Indication for ablation

Left ventricular function

Presence of structural heart disease History of dc cardioversion

Method of ablation

Mode of pacing

Rate responsive pacing

Mode switching capacity

Use of antiarrhythmic drugs at follow up

Mean (SD) length of follow up
59.5 (14.7) years

$33(53 \%)$ male, $29(47 \%)$ female

$39(63 \%)$ paroxysmal AF, $23(37 \%)$ paroxysmal atrial flutter or tachycardia

$44(71 \%)$ in group $1,14(23 \%)$ in group $2,4(6 \%)$ in group 3

32 patients $(52 \%)$

14 patients $(23 \%)$

$50(81 \%)$ by radiofrequency, $12(19 \%)$ by direct current

$31(50 \%)$ ventricular, $31(50 \%)$ dual chamber

41 patients $(66 \%)$

15 patients $(24 \%)$

10 patients $(16 \%)$

910 (720) days

$\mathrm{AF}$, atrial fibrillation; dc, direct current.

increasing patient age at the time of ablation, and VVI pacing are predictive of the development of chronic atrial fibrillation in this patient group.

(Heart 1998;79:548-553)

Keywords: atrioventricular node ablation; pacemaker mode; cardioversion; atrial fibrillation

Patients undergoing atrioventricular node ablation for paroxysmal atrial arrhythmias require implantation of a permanent pacemaker to treat the resultant complete heart block. Choice of the most appropriate method of pacing is contentious. The British Pacing and Electrophysiology Group ${ }^{1}$ has not made specific recommendations about the optimal mode of pacing for these patients but states that "the presence of atrial activity other than chronic or frequently repetitive atrial flutter or fibrillation generally requires an atrial pacemaker electrode," and by inference this supports the use of dual chamber systems in patients with paroxysmal atrial arrhythmias. Dual chamber pacing has the advantage of maintaining atrioventricular synchrony, but with the development of atrial fibrillation the atrial arm of the system becomes redundant, leading to de facto ventricular pacing. This may not, however, be a common problem, and one retrospective study has suggested that in the majority of these patients dual chamber pacing is maintained during follow up. ${ }^{2}$ Disadvantages of dual chamber systems are the increased cost and complexity of implantation and follow up, the failure rate of atrial leads, ${ }^{34}$ and the possibility of a rapid ventricular response during atrial arrhythmias.

In contrast, ventricular pacing systems are simpler to implant and follow up, but can cause pacemaker syndrome ${ }^{5}$ during sinus rhythm and may also increase the rate of progression to chronic atrial fibrillation. There is evidence that this occurs in patients receiving pacemakers for sick sinus syndrome ${ }^{6-12}$ or complete heart block, ${ }^{13}$ and patients undergoing ablation for paroxysmal atrial arrhythmias have similarities to both of these groups.

Theoretically the ideal mode of pacing for this group of patients is a dual chamber, mode switching device, ${ }^{14}$ which will maintain atrioventricular synchrony during sinus rhythm but switch to a ventricular mode during paroxysmal or chronic atrial fibrillation. Universal use of these advanced pacing systems does not, however, make the best use of resources if a high proportion of the patients develop chronic 


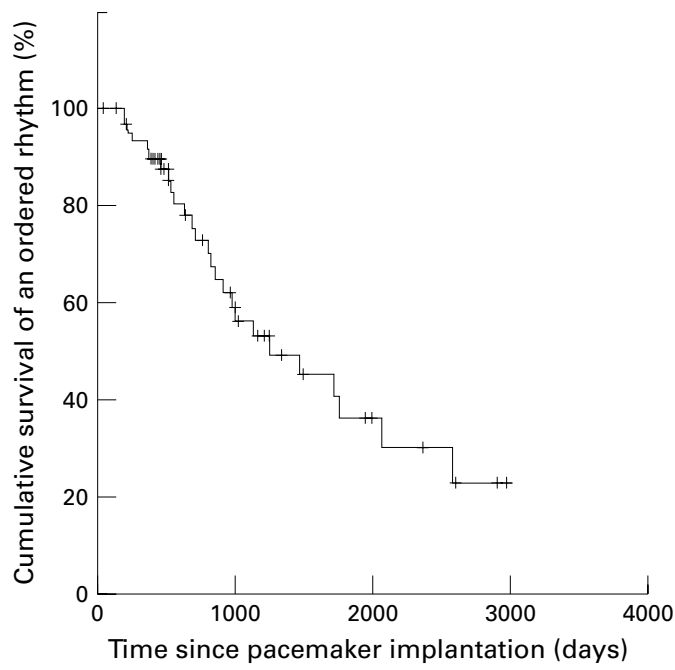

\begin{tabular}{|c||c|c|c|c|c|c|c|}
\hline $\begin{array}{c}\text { Length of follow } \\
\text { up (days) }\end{array}$ & 0 & 500 & 1000 & 1500 & 2000 & 2500 & 3000 \\
\hline $\begin{array}{c}\text { Number of patients } \\
\text { remaining in the } \\
\text { analysis }\end{array}$ & 62 & 39 & 21 & 10 & 6 & 4 & 0 \\
\hline
\end{tabular}

Figure 1 Survival of an ordered rhythm after atrioventricular node ablation. + indicates a censored event - that is, mode change or end of follow up.

atrial fibrillation and so require only a rate responsive ventricular (VVIR) pacing unit. Accurate prediction of those patients most likely to develop chronic atrial fibrillation would allow improved resource allocation. We undertook this study to assess the natural history of patients paced after atrioventricular node ablation for paroxysmal atrial arrhythmias and to test whether pacing mode is associated with the development of chronic atrial fibrillation.

\section{Methods}

We undertook a retrospective analysis of consecutive patients who have undergone complete atrioventricular node ablation for refractory paroxysmal atrial arrhythmias in this hospital between 1988 and July 1996. Sixty five were identified from the pacing department database and electrophysiology records. Patients' records were reviewed to assess age at implantation, method of ablation (by direct current (dc) or radiofrequency energy), indication for ablation (paroxysmal atrial fibrillation, paroxysmal atrial flutter, or paroxysmal atrial tachycardia), mode of pacing (DDD versus VVI, rate responsiveness, and mode switching), length of follow up, use of antiarrhythmic drugs during follow up, history of dc cardioversion before ablation, and atrial rhythm on electrocardiographic (ECG) follow up. An assessment of left ventricular function at the time of pacemaker implantation was made on the basis of ECG data, descriptions of exercise capacity, and drug lists. Left ventricular function was then divided empirically into three categories: (1) normal function; (2) impaired function; and (3) severely impaired function. The presence or absence of underlying structural heart disease (left ventricular impairment, valve disease, old myocardial infarction, or cardiomyopathy) was determined from the physical examination and echocardiographic findings at the time of implantation. Age and length of follow up were treated as continuous variables, all other variables were analysed as categorical variables. For patients not followed up at this hospital, or with no ECG recorded after August 1996, a maximum of two letters were sent to the patient's physician and local pacing department asking for information, or to the patients themselves, inviting them to attend the hospital for a 12 lead ECG.

The most recent ECGs were reviewed and the atrial rhythm classified as either ordered (sinus or atrially paced) or disordered (atrial fibrillation, flutter, or tachycardia). Follow up was censored once patients were deemed to be in a chronically disordered atrial rhythm or if the pacemaker mode was changed. Chronic disorder of atrial rhythm ("chronic atrial fibrillation") was diagnosed on the basis of either two consecutive ECGs showing atrial fibrillation, atrial flutter, or atrial tachycardia, or sustained arrhythmia on a 24 hour Holter recording, with no subsequent ordered rhythm. The two outcome measures chosen were therefore (1) a disordered atrial rhythm on the most recent ECG, and (2) chronic atrial fibrillation (as defined above).

Table 2 Univariate analyses of patient variables compared with the two outcome measures

\begin{tabular}{|c|c|c|c|c|}
\hline \multirow[b]{2}{*}{ Variable } & \multicolumn{2}{|l|}{ Disordered final ECG } & \multicolumn{2}{|l|}{ Chronic AF } \\
\hline & $\begin{array}{l}\text { Patients reaching the } \\
\text { outcome measure }\end{array}$ & $p$ value & $\begin{array}{l}\text { Patients reaching the } \\
\text { outcome measure }\end{array}$ & $p$ value \\
\hline History of dc cardioversion & $11 / 14$ & 0.015 & $10 / 14$ & 0.011 \\
\hline VVI pacing & $18 / 31$ & 0.20 & $17 / 31$ & 0.039 \\
\hline Age & & 0.011 & & 0.049 \\
\hline Antiarrhythmic drugs & $3 / 10$ & 0.17 & $2 / 10$ & 0.13 \\
\hline Structural heart disease & $20 / 32$ & 0.04 & $16 / 32$ & 0.18 \\
\hline Male sex & $17 / 33$ & 0.80 & $16 / 33$ & 0.27 \\
\hline Rate responsive pacing & $21 / 41$ & 0.79 & $19 / 41$ & 0.33 \\
\hline Normal left ventricular function & $22 / 44$ & 0.53 & $17 / 44$ & 0.37 \\
\hline Impaired left ventricular function & $8 / 14$ & 0.53 & $8 / 14$ & 0.37 \\
\hline Severely impaired left ventricular function & $1 / 4$ & 0.53 & $1 / 4$ & 0.37 \\
\hline Mode switching pacing & $9 / 15$ & 0.37 & $5 / 15$ & 0.44 \\
\hline Paroxysmal $\mathrm{AF}$ as indication for ablation & $20 / 39$ & 0.79 & $15 / 39$ & 0.47 \\
\hline $\mathrm{RF}$ as method of ablation & $25 / 50$ & 1.00 & $20 / 50$ & 0.53 \\
\hline Length of follow up & & 0.78 & & 0.70 \\
\hline
\end{tabular}

$\mathrm{AF}$, atrial fibrillation; dc, direct current; $\mathrm{RF}$, radiofrequency. 
Table 3 Multiple logistic regression of patient variables

\begin{tabular}{|c|c|c|c|c|}
\hline \multirow[b]{2}{*}{ Variable } & \multicolumn{2}{|c|}{ Disordered final ECG } & \multicolumn{2}{|l|}{ Chronic AF } \\
\hline & Odds ratio & $95 \% C I$ & Odds ratio & $95 \% C I$ \\
\hline History of dc cardioversion & 5.7 & 1.1 to 28.7 & 7.6 & 1.6 to 34.3 \\
\hline Age (years) & 1.1 & 1.0 to 1.12 & 1.04 & 1.0 to 1.1 \\
\hline VVI pacing & & & 3.2 & 0.98 to 10.5 \\
\hline
\end{tabular}

$\mathrm{AF}$, atrial fibrillation; dc, direct current.

\section{STATISTICS}

Patient groups were compared using MannWhitney $U$ tests for continuous variables and $\chi^{2}$ (or Fisher's exact) tests for categorical variables. Variables were then assessed by forward stepwise multiple logistic regression analysis. The maintenance of sinus rhythm was assessed by Kaplan-Meier survival curves and the significance of variables assessed by the log-rank test. Statistical significance was assumed at a probability (p) of $<0.05$.

\section{Results}

Of 65 patients identified three were excluded from the study - two died before hospital discharge after pacemaker implantation (one from a cardiac arrest and the other from a pulmonary embolism) and the third was lost to follow up. The study group therefore comprised 62 patients. Their baseline characteristics are summarised in table 1 . Continuous variables are expressed as mean (standard deviation).

Four of the patients initially paced in VVI mode subsequently had their pacemakers

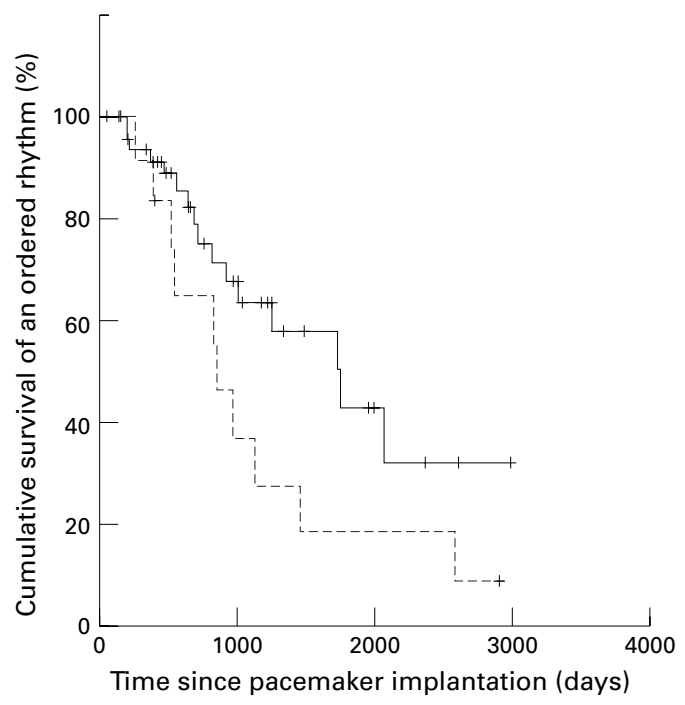

\begin{tabular}{|c||c|c|c|c|c|c|c|}
\hline $\begin{array}{c}\text { Length of follow } \\
\text { up (days) }\end{array}$ & 0 & 500 & 1000 & 1500 & 2000 & 2500 & 3000 \\
\hline $\begin{array}{c}\text { Number of patients } \\
\text { remaining with no } \\
\text { history of dc } \\
\text { cardioversion }\end{array}$ & 48 & 30 & 16 & 8 & 4 & 2 & 0 \\
\hline $\begin{array}{c}\text { Number of patients } \\
\text { remaining with a } \\
\text { history of dc } \\
\text { cardioversion }\end{array}$ & 14 & 9 & 4 & 2 & 2 & 2 & 0 \\
\hline
\end{tabular}

Figure 2 Survival of an ordered rhythm by history of $d c$ cardioversion. + indicates a censored event, that is, mode change or end of follow up; - , patients with no history of dc cardioversion; - - - - , patients with a history of dc cardioversion. upgraded to DDD (by implantation of a new system in three, and by reprogramming a dual chamber pacemaker that had been initially programmed to VVI mode in the fourth). These four patients were analysed on "an intention to treat" basis - that is, as part of the VVI group - and their follow up was censored at the point of pacemaker upgrade. Dual chamber pacemakers were routinely programmed DDD with a rate of 60 to 120 beats/ min. Ventricular pacemakers were programmed VVI at a rate of 60 or VVIR with a rate responsive range of 60 to 130 beats $/ \mathrm{min}$. There were four late deaths (at 195, 200, 360, and 532 days after pacemaker implantation).

At a mean follow up of 910 days ( 30 months) 31 patients $(50 \%)$ had a disordered atrial rhythm on their last ECG, and $26(42 \%)$ were in chronic atrial fibrillation. The survival of an ordered rhythm was assessed by Kaplan-Meier survival analysis (fig 1) and the median (SEM) survival of sinus rhythm after ablation was 1254 (383) days (42 (13) months). By 2584 days (86 months) after ablation $75 \%$ of patients were in chronic atrial fibrillation. The influence of baseline patient characteristics on the two outcome measures was assessed by univariate analysis (table 2). This shows that increasing age, previous dc cardioversion, and the presence of structural heart disease were all associated with a disordered rhythm on the final ECG and that increasing age, previous dc cardioversion, and VVI pacing were all associated with the development of chronic atrial fibrillation.

All variables were then entered into a multivariate logistic regression analysis (table 3 ). This shows that a history of dc cardioversion and increasing patient age were associated with both of the outcome measures. A history of dc cardioversion was associated with a 5.7-fold increase in the risk of a disordered rhythm on last follow up and a 7.6-fold risk of developing chronic atrial fibrillation. Despite this high odds ratio, a history of dc cardioversion just failed to divide the population into significantly different groups on survival analysis (fig 2), $\mathrm{p}=$ 0.08 . Increasing patient age was associated with an increase in risk of $10 \%$ per year of a disordered atrial rhythm on the final ECG and of $4 \%$ per year of developing chronic atrial fibrillation. VVI pacing was significantly associated with progression to chronic atrial fibrillation after the effects of age and dc cardioversion had been entered into the regression models - that is, their inclusion in the multiple regression model significantly improved the model's ability to predict chronic atrial fibrillation. Although the odds ratio for this association is greater than 3, the $95 \%$ confidence interval spans 1 . The magnitude of the association between VVI pacing and the development of chronic atrial fibrillation therefore fails to reach significance. Mode of pacing did not significantly alter the maintenance of sinus rhythm on survival analysis, $\mathrm{p}=0.15$ (fig 3 ). No other variables examined related to outcome. 


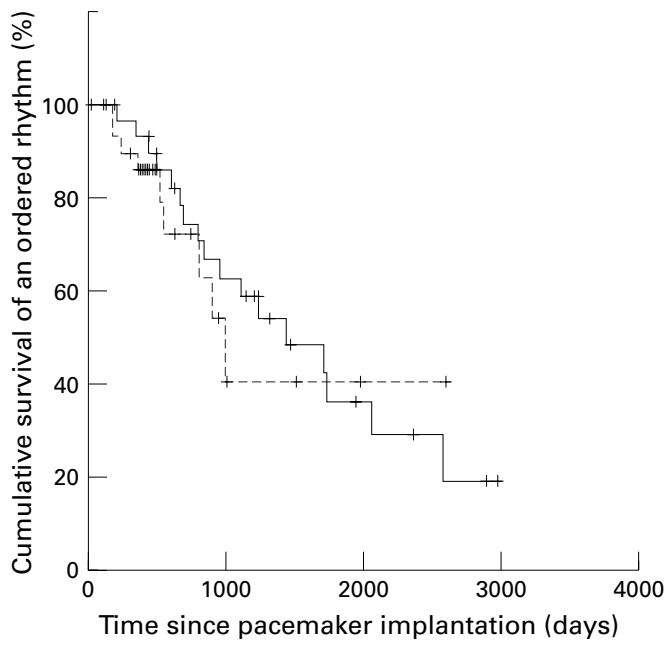

\begin{tabular}{|c||c|c|c|c|c|c|c|}
\hline $\begin{array}{c}\text { Length of follow } \\
\text { up (days) }\end{array}$ & 0 & 500 & 1000 & 1500 & 2000 & 2500 & 3000 \\
\hline $\begin{array}{c}\text { Number of patients } \\
\text { remaining paced } \\
\text { VVI }\end{array}$ & 31 & 25 & 16 & 8 & 5 & 3 & 0 \\
\hline $\begin{array}{c}\text { Number of patients } \\
\text { remaining paced } \\
\text { DDD }\end{array}$ & 31 & 14 & 4 & 2 & 1 & 1 & 0 \\
\hline
\end{tabular}

Figure 3 Survival of an ordered rhythm by mode of pacing. + indicates a censored event - that is, mode change or end of follow up. -, patients paced in DDD mode; - - - , patients paced in VVI mode.

\section{Discussion}

In this analysis we show that patients undergoing atrioventricular node ablation for paroxysmal atrial arrhythmias are at high risk of developing chronic atrial fibrillation- $42 \%$ had developed chronic atrial fibrillation after a mean follow up of 30 months. Increasing patient age at pacemaker implantation, a history of dc cardioversion, and VVI pacing are all associated with the development of chronic atrial fibrillation. No other variables, including the use of rate responsive pacing or the method of atrioventricular node ablation, were associated with outcome.

The only other published study to have looked specifically at this patient group ${ }^{2}$ retrospectively assessed the atrial rhythm of 49 patients after atrioventricular node ablation for paroxysmal atrial arrhythmias. At a mean (SD) follow up of 18.6 (15.6) months, $67 \%$ had either sinus rhythm or atrial pacing (an ordered rhythm by our definition). This figure is compatible with our findings: $50 \%$ of patients had an ordered rhythm at 30 (24) months. The smaller proportion of patients in our study with an ordered rhythm possibly reflects their longer follow up, although increased length of follow up was not independently associated with an increased risk of a disordered final ECG. Mitchell et al argue that as only $14 \%$ of their patients had an atrial arrhythmia on every follow up ECG, the development of chronic atrial fibrillation was relatively infrequent. ${ }^{2}$ However, $25 \%$ of their patients had both atrial arrhythmias and sinus rhythm documented during follow up and it is not clear what proportion of these developed chronic atrial fibrillation by our definition. A theoretical maximum of 33\% of the whole study group could have developed chronic atrial fibrillation during their follow up period and this is similar to the $42 \%$ of our patient group with chronic atrial fibrillation.

Why should these patients develop chronic atrial fibrillation? Age is a well recognised risk factor for the development of atrial fibrillation in the general population, ${ }^{15}$ in patients paced for sick sinus syndrome, ${ }^{16}$ and in patients undergoing coronary artery bypass grafting. ${ }^{17} 18$ It is not surprising therefore that increasing age was associated with an additional risk of chronic atrial fibrillation in our study.

A history of paroxysmal atrial arrhythmias is also a recognised risk factor. Although little is known about the natural history of patients with paroxysmal atrial arrhythmias who do not receive pacemakers, it has recently been suggested that $25 \%$ to $67 \%$ of patients with paroxysmal atrial fibrillation will eventually develop chronic atrial fibrillation. ${ }^{19}$ The data on patients with paroxysmal atrial arrhythmias who require pacemaker implantation are more extensive. Several studies of paced patients have shown that a history of paroxysmal atrial arrhythmias markedly increases the risk of developing chronic atrial fibrillation. In a retrospective study Gross et al found that 37\% of patients with a history of paroxysmal atrial arrhythmias, but only $9 \%$ without, developed atrial fibrillation requiring a change of pacing mode from DDD to VVI over a mean follow up period of 33 months. ${ }^{20}$ In a larger study ${ }^{16}$ of 507 patients paced for sick sinus syndrome, multivariate analysis showed that a history of paroxysmal atrial arrhythmias increased the risk of progression to chronic atrial fibrillation more than 16-fold. In the largest prospective study of patients paced for sick sinus syndrome it was found that the only independent predictor of developing chronic atrial fibrillation after a mean follow up of 3.3 years was a history of paroxysmal atrial arrhythmias, and at a mean of eight years, a history of paroxysmal atrial arrhythmias and the left ventricular end diastolic diameter at the time of randomisation. ${ }^{621}$ This finding has been reproduced in the preliminary results of the PASE study ${ }^{22}$ (comparing VVIR with DDDR pacing for patients with sick sinus syndrome or complete heart block), in which a history of previous atrial fibrillation or other supraventricular tachycardia was the only factor predictive of the development of chronic atrial fibrillation in 403 patients with a mean follow up of 19.5 months.

In a subgroup analysis of 375 patients with a history of paroxysmal atrial arrhythmias being paced for sick sinus syndrome, length of history and prolonged episodes of paroxysmal atrial arrhythmias were the most significant predictors of chronic atrial fibrillation. ${ }^{16}$ Use of antiarrhythmic drugs before implantation was also an independent predictor of chronic atrial fibrillation. We have shown that a history of dc cardioversion conferred a sevenfold risk of developing chronic atrial fibrillation; it might therefore be a marker of more prolonged or severe episodes of paroxysmal atrial arrhythmias, but it is more likely that it is a marker of 
failure of spontaneous conversion of atrial fibrillation to sinus rhythm and thus of persistent rather than paroxysmal atrial fibrillation. Patients with persistent atrial fibrillation (defined by Sopher and $\mathrm{Camm}^{23}$ as atrial fibrillation which fails to undergo spontaneous cardioversion, but which can be converted electrically or chemically) who undergo atrioventricular node ablation are unlikely to undergo dc cardioversion after their ablation and thus will remain in atrial fibrillation. Their rhythm may then be diagnosed as chronic atrial fibrillation, although a more accurate description might be untreated persistent atrial fibrillation.

An alternative explanation for the association between dc cardioversion and chronic atrial fibrillation is that a need for dc cardioversion is a marker of structural heart disease which could increase the severity of symptoms caused by episodes of atrial fibrillation. Cardiac failure is strongly associated with the development of atrial fibrillation ${ }^{1524}$ in the general population, but in our group there was no association between structural heart disease, including left ventricular dysfunction, and the development of chronic atrial fibrillation on multivariate analysis, despite the association between it and a disordered atrial rhythm on the final ECG on univariate analysis.

In keeping with evidence of the detrimental effect of VVI pacing on progression to chronic atrial fibrillation in other patients with paroxysmal atrial arrhythmias, ${ }^{76}{ }^{25}$ VVI pacing in this study was associated with an increased risk of chronic atrial fibrillation. This is consistent with our preliminary analysis of a subset of the same patient group, ${ }^{26}$ which suggested that DDD pacing protects against the development of atrial fibrillation. Whether mode of pacing influences the rate of progression to chronic atrial fibrillation in patients with paroxysmal atrial arrhythmias who have undergone atrioventricular node ablation has not previously been studied systematically. Observations from large series of patients with sick sinus syndrome suggest that in patients with a history of paroxysmal atrial arrhythmias, ventricular pacing is associated with a prevalence of atrial fibrillation of 53-63\% compared with $11-29 \%$ for physiological pacing. ${ }^{7625}$ Before ablation, patients with paroxysmal atrial arrhythmias are similar to patients with sick sinus syndrome with tachy-brady syndrome. Although postablation they are unlikely to have intact retrograde atrioventricular nodal conduction during ventricular pacing (which has been suggested as a further risk factor for developing chronic atrial fibrillation ${ }^{12}$ ), they will still experience the adverse haemodynamic consequences of loss of atrioventricular synchrony that possibly enhances the progression to chronic atrial fibrillation.

Mode of pacing may also have different effects in patients with paroxysmal and persistent atrial fibrillation. If dual chamber pacing protects against the development of atrial fibrillation, this protection will be greater in patients with paroxysmal rather than persistent atrial fibrillation, as these patients will spend more time paced in dual chamber mode. This may reduce the tendency to develop chronic atrial fibrillation. The importance of VVI pacing in the development of chronic atrial fibrillation remains contentious, however, ${ }^{27}$ and is currently the subject of large scale prospective studies for patients with sick sinus syndrome. ${ }^{28} 29$

The association of dc cardioversion with an increased risk of chronic atrial fibrillation may allow the use of more appropriate pacing modes. Our study suggests that patients who have previously required dc cardioversion for an episode of atrial fibrillation have a sevenfold greater risk of progressing to chronic atrial fibrillation than those who have not. Such patients might therefore best be treated - as has been previously recommended ${ }^{30}$ - with a VVIR pacing system. The choice of a VVI pacemaker for these patients increases the risk of chronic atrial fibrillation, but DDD pacing does not significantly prolong the survival of sinus rhythm. Patients who have not required dc cardioversion, because they have always reverted spontaneously to sinus rhythm before ablation, would gain haemodynamic advantages from dual chamber pacing and are likely to maintain this pacing mode for longer periods of time.

A further implication of these data is that all these patients are at a high risk of progression to chronic atrial fibrillation. As most of these patients have undergone atrioventricular node ablation because of failure of or intolerance to antiarrhythmic drugs, it is unlikely that continuing use of drug treatment after ablation would be successful. Attempts to maintain sinus rhythm in these patients may therefore be misplaced, and management should focus on optimising ventricular rate control and appropriate anticoagulation to minimise risks of thromboembolism.

\section{LIMITATIONS}

Any retrospective study has inherent limitations. There is potential for bias between the subject groups for which it may not be possible to control. We assessed left ventricular function and structural heart disease retrospectively from case notes and may have underestimated the prevalence of left ventricular dysfunction, or of heart disease. The end points of the study were derived from review of ECGs recorded at variable intervals. Different treatment strategies reflect advances in ablation and pacemaker technology since 1988 . Since that time standard practice has progressed from dc to radiofrequency ablation techniques and from VVI to complex mode switching, rate responsive, dual chamber pacemakers. These technological advances have altered opinions concerning both patient selection and subsequent optimal pacing mode.

\section{CONCLUSIONS}

Survival analysis of atrial rhythm in patients undergoing atrioventricular node ablation for paroxysmal atrial arrhythmias suggests that over a prolonged period of follow up the vast majority progress to chronic atrial fibrillation. Risk factors for this are a history of dc cardioversion, increasing patient age, and VVI 
pacing. Patients who have required dc cardioversion before atrioventricular node ablation appear to gain little from the implantation of a dual chamber pacing system.

GMG is supported by a grant from the British Heart Foundation. We are indebted to Sarah Roberts, Department of Statistics, University of Newcastle upon Tyne for statistical advice.

1 The Working Party of the British Pacing and Electrophysiology Group. Recommendations for pacemaker prescription for symptomatic bradycardia. Br Heart F 1991;66:185-91.

2 Mitchell MA, Ackerman SJ, Nath S, et al. Atrial rhythm after atrioventricular junctional ablation. Am $\mathcal{f}$ Cardiol 1996;78:1251-4.

3 Aggarwal RK, Connelly DT, Ray SG, et al. Early complications of permanent pacemaker implantation: no difference between dual and single chamber systems. Br Heart $f$ 1995;73:571-5.

4 Chauhan A, Grace AA, Newell SA, et al. Early complications after dual chamber versus single chamber pacemaker implantation. PACE 1994;17:2012-15.

5 Travill CM, Sutton R. Pacemaker syndrome: an iatrogenic condition. Br Heart 7 1992;68:163-6.

6 Andersen HR, Thuesen L, Bagger JP, et al. Prospective randomised trial of atrial versus ventricular pacing in domised trial of atrial versus ventricular
sick-sinus syndrome. Lancet 1994;344:1523-8.

7 Sick-sinus syndrome. Lancet 1994;344:1523-8. Hesselson AB, Parsonnet V, Bernstein AD, et al. Deleterious
effects of long-term single-chamber ventricular pacing in effects of long-term single-chamber ventricular pacing in patients with sick sinus syndrome: the hidden benefits of
dual-chamber pacing. F Am Coll Cardiol 1992;19:1542-9.

8 Rosenqvist M, Brandt J, Schuller H. Long-term pacing in sinus node disease: effects of stimulation mode on cardiovascular morbidity and mortality. Am Heart $\mathcal{f}$ 1988;116:16-22.

9 Santini M, Alexidou G, Ansalone G, et al. Relation of prognosis in sick sinus syndrome to age, conduction defects and modes of permanent cardiac pacing. Am 7 Cardiol 1990;65: 729-35.

10 Sutton R, Kenny RA. The natural history of sick sinus syndrome. PACE 1986;9:1110-14.

11 Hofgartner F, Maier B, Eisele R, et al. Pacemaker therapy for the sick sinus node syndrome. Does the atrially involved pacemaker system lower the frequency of atrial fibrillation pacemaker system lower the frequency of atrial fibrillation and thromboembolic complications as

12 Egabosti A, Gueunoun M, Saadijan A, et al. Long-term follow-up of patients treated with VVI pacing and sequential pacing with special reference to VA retrograde conduction. PACE 1988;11:1929-34.

13 Feuer JM, Shandling AH, Messenger JC. Influence of cardiac pacing mode on the long-term development of atrial fibrillation. Am $\mathcal{F}$ Cardiol 1989;64:1376-9.
14 Kamalvand $\mathrm{K}$, Tan $\mathrm{K}$, Kotsakis A, et al. Is mode switching beneficial? A randomized study in patients with paroxysmal atrial tachyarrhythmias. F Am Coll Cardiol 1997;30:496504.

15 Kannel W, Abbott R, Savage D, et al. Epidemiological features of atrial fibrillation. The Framingham study. $N$ Engl f Med 1982;306:1018-22.

16 Sgarbossa EB, Pinski SL, Maloney JD, et al. Chronic atrial fibrillation and stroke in paced patients with sick sinus syndrome. Relevance of clinical characteristics and pacing modalities. Circulation 1993;88:1045-53.

17 Creswell LL, Schuessler RB, Rosenbloom M, et al. Hazards of postoperative atrial arrhythmias. Ann Thorac Surg 1993; 56:539-49.

18 Fuller JA, Adams GG, Buxton B. Atrial fibrillation after coronary artery bypass grafting. Is it a disorder of the elderly? 7 Thorac Cardiovasc Surg 1989;97:821-5.

19 Aboaf AP, Wolf PS. Paroxysmal atrial fibrillation. A common but neglected entity. Arch Intern Med 1996;156: 362-7.

20 Gross JN, Moser S, Benedek ZM, et al. DDD pacing mode survival in patients with a dual-chamber pacemaker. $f \mathrm{Am}$ Coll Cardiol 1992;19:1536-41.

21 Andersen HR, Nielson JC, Bloch Tomsen PE, et al. Long-term follow up of patients from a randomised trial of atrial versus ventricular pacing for sick sinus syndrome. Lancet 1997;350:1210-16.

22 Stambler B, Ellenbogen K, Pinsky S, et al. Development of post-implant atrial fibrillation during DDDR versus VVIR pacing in the PASE trial. PACE 1996;19:619.

23 Sopher SM, Camm AJ. Atrial fibrillation: maintenance of sinus rhythm versus rate control. Am f Cardiol 1996;77:24$37 \mathrm{~A}$.

24 Krahn A, Manfreda J, Tate R, et al. The natural history of atrial fibrillation: incidence, risk factors, and prognosis in the Manitoba follow up study. Am $\mathcal{F}$ Med 1995;98:476-84.

25 Vanerio G, Maloney JD, Pinski SL, et al. DDIR versus VVIR pacing in patients with paroxysmal atrial tachyarrhythmias. PACE 1991;14:1630-8.

26 Gribbin GM, Bourke JP, McComb JM. Dual versus single chamber pacemakers in patients with paroxysmal atrial fibrillation treated with atrioventricular node ablation. PACE 1997;20:1592.

27 Lamas GA, Estes NM, Schneller S, et al. Does dual chamber or atrial pacing prevent atrial fibrillation? The need for a randomized controlled trial. PACE 1992;15:1109-13.

28 Charles RG, McComb JM. Systematic trial of pacing to prevent atrial fibrillation. Heart 1997;78:224-5.

29 Lamas GA. Pacemaker mode selection and survival: a plea to apply the principles of evidence based medicine to cardiac pacing practice. Heart 1997;78:218-20.

30 Jensen SM, Bergfeldt L, Rosenqvist M. Long-term follow-up of patients treated by radiofrequency ablation of the atrioventricular junction. PACE 1995;18:1609-14. 